\title{
Application of Variable Universe Fuzzy Control PID in Temperature Command of the Animal Building
}

\author{
Xiao $\mathrm{Yu}^{1,2}$, Hai-Ye $\mathrm{Yu}^{1}$ and Guo-Gang $\mathrm{Zhao}^{1}$ \\ ${ }^{1}$ School of Biological and Agricultural Engineering, Jilin University, Changchun, \\ China \\ ${ }^{2}$ School of Electrical and Information Engineering, Northeast Agricultural University, \\ Harbin, China \\ neaufish@163.com
}

\begin{abstract}
Temperature is a universe and significant data in production and scientific experiment, effective measurement and control of the temperature are widely applied in every part of national economy. Therefore, temperature control has great significance; relevant requirements regarding temperature control are also higher and higher.

Temperature command system for animal house is a complex system with features of time varying, nonlinear, inaccurate model and unknown operating circumstance, temperature controlled by air-conditioner is less accurate and will affect the stability of the system performance. Regarding existing problems of long responding time, less stability and other disadvantages of the conventional PID control, we hereby raise a new approach of variable universe fuzzy PID control which is characterized with quick responding speed, better stability and stronger anti-interruption performance. Through the application of variable universe fuzzy control theory, which combined with the advantages of conventional PID and variable universe fuzzy control, forming the calculation of self-applied variable universe fuzzy control PID. Improve the accuracy of fuzzy inference by variable universe control theory, and realize the online tuning of PID data through fuzzy inference. Stimulate on Matlab, relevant stimulation results revealed that the control ways is clearly superior than conventional PID control, therefore effectively improve the dynamic property, stability precision and robustness, the system has better future of industrial application.
\end{abstract}

Keywords: Nonlinear, Variable Universe, Fuzzy Control, Animal House, Environmental Factors

\section{Introduction}

Circumstance control system of animal house is a complex system, controlled volume include numbers of environmental factors inside the animal house, temperature have great influence on animal growth and generation of noxious gas, therefore, temperature control is the priority of the circumstance control system of animal house. During the process of circumstance control of the animal house, because circumstance of animal house is a multivariable non-linear system and is difficult to form mathematic model, although there is no need to form accurate mathematic model through fuzzy control, but the selection of initial universe have tremendous influence on the accuracy of the whole control system. Therefore, variable universe fuzzy control can solve the problem with the selection of the initial universe. 
Conventional PID control is featured with simple, reliable, floating and other advantages, it is a widely used and relatively classic control approach concluded through the process control, however, it can achieve expected good effects which can hardly achieved by other systems characterized with non-linear and uncertainty; The integral separation PID control raised in bibliography [1] has improved greatly when compared with conventional PID control in aspect of overshoot, however, its effect on dynamic control of non-linear system is still not satisfied enough; Self-adaptive fuzzy PID control raised in bibliography [2] is a combination of fuzzy control and conventional PID control, which can not only meet the requirements of controlled objects' non-linear and inaccurate mathematic model, but also has advantages of PID control such as simple structure, high controlling accuracy and good control effective. Regarding problem of improvement of accuracy of fuzzy control under the condition of keeping regulation, bibliography [3-4] firstly raised variable universe theory; under the condition of keeping regulations, universe is shrinking with the decreasing of tolerance. The thesis introduce variable universe theory on the basis of self-adaptive fuzzy PID control powered by tolerances, raised a new variable universe fuzzy control PID. The thesis made detailed discussion regarding fuzzy interpolation theory, and made stimulation test and comparison for conventional PID control, integral separation PID control, conventional fuzzy PID control and variable universe PID control under the condition of Matlab stimulation circumstance. The result of stimulation shows that the variable universe fuzzy control PID has better performances than the above three mentioned conventional approach in aspect of responding speed, controlling accuracy and stability.

\section{Variable Universe Fuzzy Control}

\subsection{Fuzzy Control Principle}

Fuzzy control is the application of fuzzy logical theory in control engineering. Its basic principle is to use the language to summarize the operators' control strategy, and to use linguistic variables and fuzzy set theory format the control algorithm. Fuzzy control principle is shown as Figure 1. Design of fuzzy control includes three basic processes: fuzzy - fuzzy inference - non-fuzzy treatment.

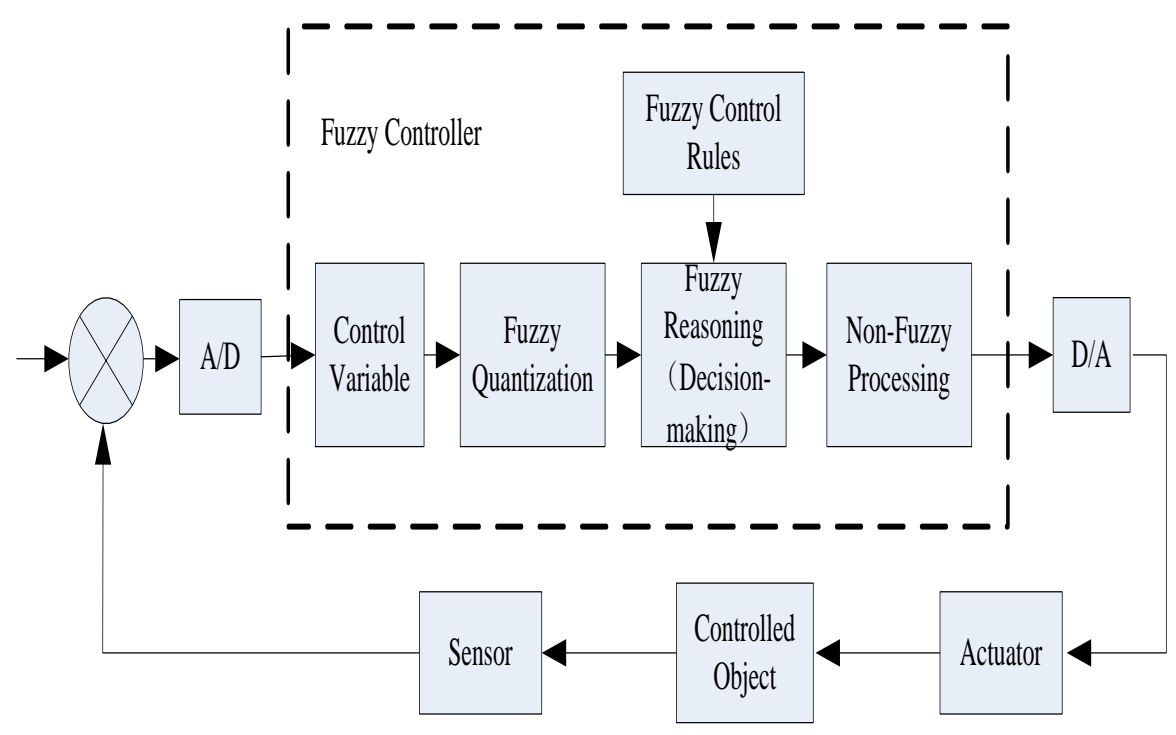

Figure 1. Fuzzy Control Principle 


\subsection{Theory of Variable Universe Fuzzy Control}

During the process of temperature control of the animal house, it is difficult to set up relevant mathematic model since the temperature control is a multivariable non-linear system, although fuzzy control do not need to set up accurate mathematic model but the selection of the initial universe have great influence on the accuracy of the complete control. Therefore, the problem of universe selection can be solved through variable universe fuzzy control. The system will take temperature control in animal house as an example; hence explain the actual application of the variable universe fuzzy control technology in temperature control of the animal house [5-7].

Variable universe is shrinked with the decrease of tolerance and vice versa under the condition of the same fuzzy classification of the universe. Transform the initial regulation base concluded by experts into more effective new regulation base through the transformation of the universe. Generally speaking, there is no variant on the number of regulation; however, since the shrinkage of the universe and detail the part of regulation, which equals to increase the number of regulations, meanwhile increase the control accuracy [8].

After the raising of the variable universe theory, relevant modified designation ways were also raised. Figure 1 showed the initial universe and its fuzzy control. Set the initial universe of $\mathrm{e}_{1}$ as [-E, E], E is the real number, according to the 7 rules, classify [-E, E] as the Figure 1 showed. As the control goes, relevant tolerance is moving toward the zero (ZE), which means the decrease of the tolerance, it is clear that the tolerance of the universe [-E, E] is bigger when compared with the shrink one. As the fuzzy control move, relevant control accuracy will be affected [9-12].

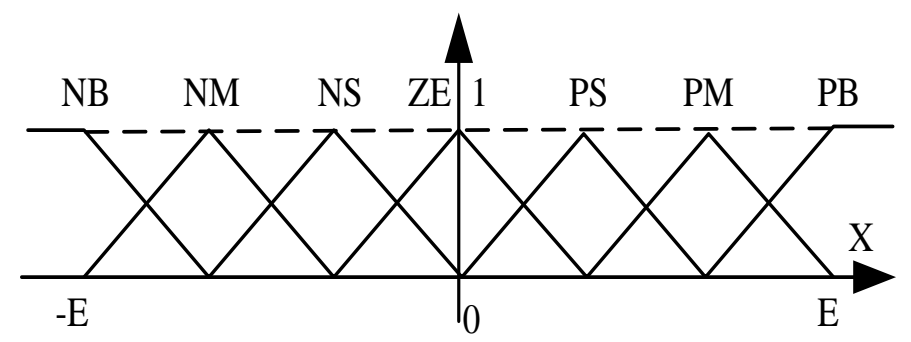

\section{Figure 2. Initial Universe and Fuzzy Partition}

Universe shrink approach is shrinking with the decrease of the tolerance of the universe under the condition of the same regulation. As the Figure 3 showed, it considered the variant process of the input variant tolerance $\mathrm{e}_{1}$. Through the "elastic and shrink" factor a(x) turning initial universe [-E, E] into $[-\alpha(x) E, \alpha(x) E]$, the $\alpha(x)$ among them is continuous function of the tolerance variant $\mathrm{x}: \alpha(\mathrm{x})[-1,1]$. 


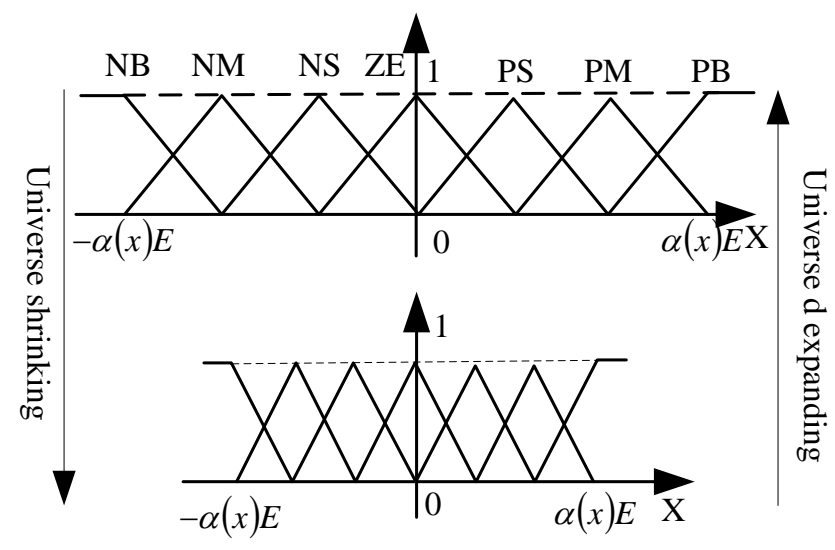

Figure 3. Universe Shrinking and Expanding

\subsection{Selection of Flexible Factors of Variable Universe Fuzzy Controller}

The key of the variable universe fuzzy controller is how to ensure the suitable system of the universe shrinkage, that is to say, ensure the adequate shrinkage factor, let the final control effect can meet the requirement with the maximum limit [13].

The flexible factor based on fuzzy regulation can describe flexible degree through the exact function. Basic theory is as follows: When the input data (Here, $\mathrm{e}_{1}$ and $\mathrm{e}_{2}$ ) increased, in order to have no effect on system increase while keep it from overshoot, the universe will be kept, when the input data decrease, the universe will be shrinked, relevant fuzzy classification defined on universe will also be compressed, hence, increase the number of available regulations, therefore eliminate the contradiction between the number of fuzzy regulations and controlling accuracy, increase the controlling sensitivity and stabling accuracy. The flexible factor based on function model will express flexible degree through some special function. Set the flexible factor of the input variant $e_{1}$ and $e_{2}$ as $\alpha_{1}$ and $\alpha_{2}$ then the regulation of the shrinking factor of the input universe based on fuzzy regulation will be as Table 1 showed.

Table 1. Input Rules of Universe Scale Factor

\begin{tabular}{cccccccc}
\hline $\mathrm{e}_{1} / \mathrm{e}_{2}$ & PB & PM & PS & ZO & NS & NM & NB \\
\hline$\alpha_{1} / \alpha_{2}$ & B & M & S & ZO & S & S & N \\
\hline
\end{tabular}

$\mathrm{B}, \mathrm{M}, \mathrm{S}, \mathrm{Z}$ showed the "expansion or shrinkage degree" of the universe. Regarding the value of the output universe flexible factor $\beta$, it should be determined by $\mathrm{e}_{1}$ and $\mathrm{e}_{2}$, that is determine the expansion or shrinkage of the output universe based on the responding condition of the system according to the $\mathrm{e}_{1}$ and $\mathrm{e}_{2}$. When the value of $\mathrm{e}_{1}$ and $\mathrm{e}_{2}$ is very big and have the same signal, it revealed that the gap between the system and the settled value is big, and the system is also move from the settled value at high speed therefore enlarge the tolerance, meanwhile there should be big controlling value to readjust the system and let it track the settled value as soon as possible. Therefore, value $\beta$ should be bigger hen expand the output universe. The above regulation should be expresses as rules follows:

If $\mathrm{e}_{1}$ is $\mathrm{PB}$ and $\mathrm{e}_{2}$ is $\mathrm{PB}$, then $\beta$ is $\mathrm{VB}$; 
If $e_{1}$ is $N B$ and $e_{2}$ is $N B$, then $\beta$ is $V B$.

When the value of $e_{1}$ and $e_{2}$ is very big but have the contradictory signal, it revealed that the gap between the system and the settled value is big, while the system is move towards the settled value at high speed, meanwhile the controlling value should be kept at certain value to guaranteed the fast track of the settled value without too much variant.

Therefore, value $\beta$ should be smaller to ensure the limited compression of the output universe. The above regulation should be expresses as rules follows:

If $e_{1}$ is $P B$ and $e_{2}$ is $N B$, then $\beta$ is $Z$;

If $e_{1}$ is $N B$ and $e_{2}$ is $P B$, then $\beta$ is $Z$.

When the value of $e_{1}$ is near zero while the value of $e_{2}$ is very big, it revealed that the gap between the system and the settled value is very small, while the system is move away from the settled value at high speed, meanwhile the controlling value should be kept at big value to keep the system from the tendency of the moving away from the settled value. Therefore, value $\beta$ should be bigger to ensure the expansion of the output universe. The above regulation should be expresses as rules follows:

If $e_{1}$ is $Z$ and $e_{2}$ is $N B$, then $\beta$ is $B$;

If $e_{1}$ is $Z$ and $e_{2}$ is $P B$, then $\beta$ is $B$.

We can infer the relevant language variant value of the value $\beta$ for $e_{1}$ and $e_{2}$ under different circumstances. The output universe flexible factor based on the fuzzy regulation is showed as Table 2 .

Table 2. Output Rules of Universe Scale Factor

\begin{tabular}{|c|c|c|c|c|c|c|c|}
\hline \multirow{2}{*}{$\mathrm{e}_{1}$} & \multicolumn{7}{|c|}{$\mathrm{e}_{2}$} \\
\hline & NB & NM & NS & $\mathrm{ZO}$ & PS & $\mathrm{PM}$ & $\mathrm{PB}$ \\
\hline NB & VB & VB & VB & B & SB & $S$ & $\mathrm{ZO}$ \\
\hline $\mathrm{NM}$ & VB & VB & $\mathrm{B}$ & B & MB & $S$ & VS \\
\hline NS & VB & MB & B & VB & VS & $S$ & VS \\
\hline $\mathrm{ZO}$ & $S$ & MB & B & VB & VS & $S$ & VS \\
\hline PS & VS & $S$ & VS & VB & B & MB & $\mathrm{VB}$ \\
\hline $\mathrm{PM}$ & VS & $S$ & MB & B & B & VB & $\mathrm{VB}$ \\
\hline PB & $\mathrm{ZO}$ & $S$ & SB & B & VB & VB & $\mathrm{VB}$ \\
\hline
\end{tabular}

\subsection{Designation of PID Controller for Variable Universe Fuzzy Control}

In the temperature control system of the variant universe fuzzy control PID, the structure of controller is as Figure 4 showed. The system is classified into upper, middle and lower in total three categories, the upper category is the modification unit of flexible factor, adjust the input of the unit through the temperature tolerance $e_{1}$ of the stripping tower and the temperature tolerance variant ratio $e_{2}$ of the animal house, get the flexible factor $\alpha_{1}(\mathrm{t}), \alpha_{2}(\mathrm{t})$ of the universe $\mathrm{e}_{1}$ and $\mathrm{e}_{2}$ through fuzzy inference, while the output universe flexible factor is $\beta(t)$; The middle category is the fuzzy control unit of variant universe, get the fixed quantity $\Delta \mathrm{K}_{\mathrm{p}}, \Delta \mathrm{K}_{\mathrm{i}}, \Delta \mathrm{K}_{\mathrm{d}}$ of the output variant PID control 
reference through fuzzy control and then plus the initial value through the addition arithmetic device then come up with the optimized PID control data $\mathrm{K}_{\mathrm{p}}, \mathrm{K}_{\mathrm{i}}, \mathrm{K}_{\mathrm{d}}$; The lower layer is the PID control unit; its output control quantity driver will execute the temperature control of the animal house [14-16].

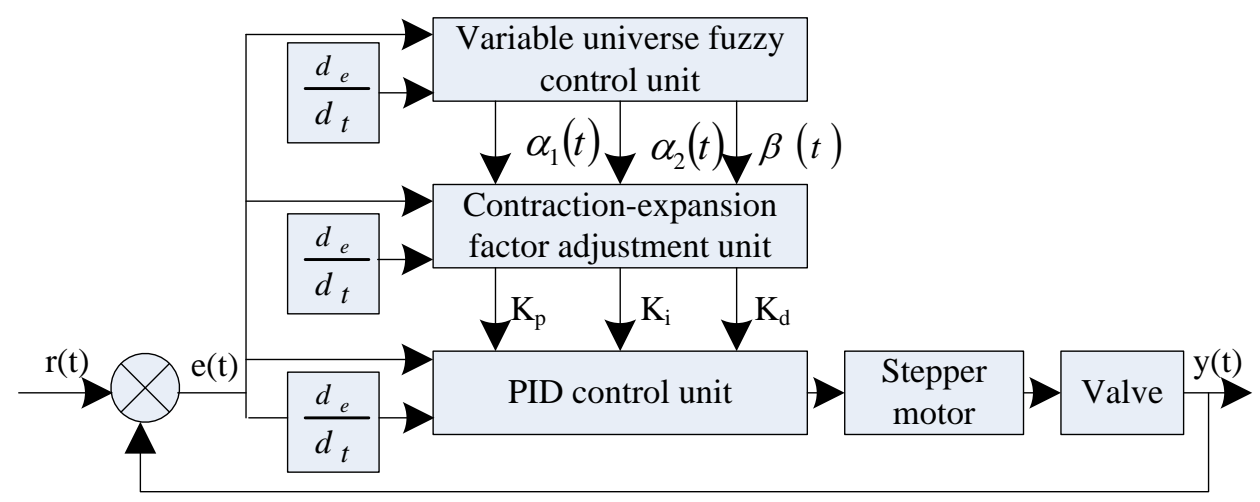

Figure 4. Variable Universe Fuzzy PID Controller Block Diagram

The variation of the temperature e inside the animal house is within the range of ($7 \sim 7)^{\circ} \mathrm{C}$, basic universe for temperature variation ratio is $(-2 \sim 2)^{\circ} \mathrm{C} / \mathrm{h}$, turn fuzzy language inference $e_{1}$ and $e_{2}$ into the integral universe $\{-6,-5,-4,-3,-2,-1,0,1,2,3,4,5,6\}$. The universe for fuzzy subset used by $\Delta \mathrm{K}_{\mathrm{p}}, \Delta \mathrm{K}_{\mathrm{i}}$ and $\Delta \mathrm{K}_{\mathrm{d}}$ is $\{-0.06,-0.05,-0.04,-0.03,-0.02,-$ $0.01,0,0.01,0.02,0.03,0.04,0.05,0.06\}$, introduce flexible factor $\alpha_{1}(\mathrm{t}), \alpha_{2}(\mathrm{t})$ according to the above data, the universe for $\mathrm{e}_{1}$ is $\alpha_{1}(\mathrm{t}) \mathrm{E}_{1}=\left[-7 \alpha_{1}(\mathrm{t}), 7 \alpha_{1}(\mathrm{t})\right]$; Universe for $\mathrm{e}_{2}$ is $\alpha_{2}(\mathrm{t})$ $\mathrm{E}_{2}=\left[-7 \alpha_{2}(\mathrm{t}), 7 \alpha_{2}(\mathrm{t})\right]$.

\section{Operational Industrial Model for the System}

The latest management \& control system developed by China adopted following temperature control theory: monitoring the temperature at the exit of ventilation tunnel by using a temperature sensor, the monitor will process and calculate on basis of the tolerance between the settled temperature and monitored temperature, output signal to the electric motor to adjust the open scale of the cold or warm ventilation entrance, hence realize the change setting of the ventilation temperature. The requirement of the animal house is to guarantee the $5{ }^{\circ} \mathrm{C}$ cold wind, $21^{\circ} \mathrm{C}$ warm wind and some other ventilation requirements according to different geographic, seasonal and environmental conditions [17-20].

The power system, warm wind temperature, cold wind temperature, environmental data, working time and other factors of the animal house have effect on the temperature at the ventilation exit, its temperature control system is a time varying, non-linear, uncertain with working environment-order inertial pure lag system, its control model is:

$$
G(x)=\frac{K e^{-\tau s}}{T s+1}
$$

In the model, $\mathrm{K} 、 \mathrm{~T} 、 \tau$ represent the open-loop gain, inertial time constant and pure lag time constant of the object model [21-23].

According to the on-site examination the model of the temperature control for animal house is: 


$$
G(x)=\frac{5 e^{-30 s}}{60 s+1}
$$

\section{Result of Stimulation}

Take the simulation of $20^{\circ} \mathrm{C}$ warm wind control generated by animal house temperature control system as the example, separately using conventional PID control, integral separation PID control, conventional fuzzy PID control and variable universe fuzzy PID control in total four approaches to conduct simulate examination. Its industrial process control transmission function is as the formula (2):

$$
G(x)=\frac{5 e^{-30 s}}{60 s+1}
$$

Figure 5 showed the simulated responding curve of the four different approaches at $20^{\circ} \mathrm{C}$ warm wind. It is clearly showed that, under the influence of the fuzzy control judgment language, variant universe fuzzy PID control and fuzzy PID control have better performance in aspect of overshoot and time adjustment, but their increasing interval is longer; Integral separation PID control is better than PID control in aspect of overshoot. The figure of overshoot for four approaches is: $2 \%, 4 \%, 11 \%$ and $30 \%$.

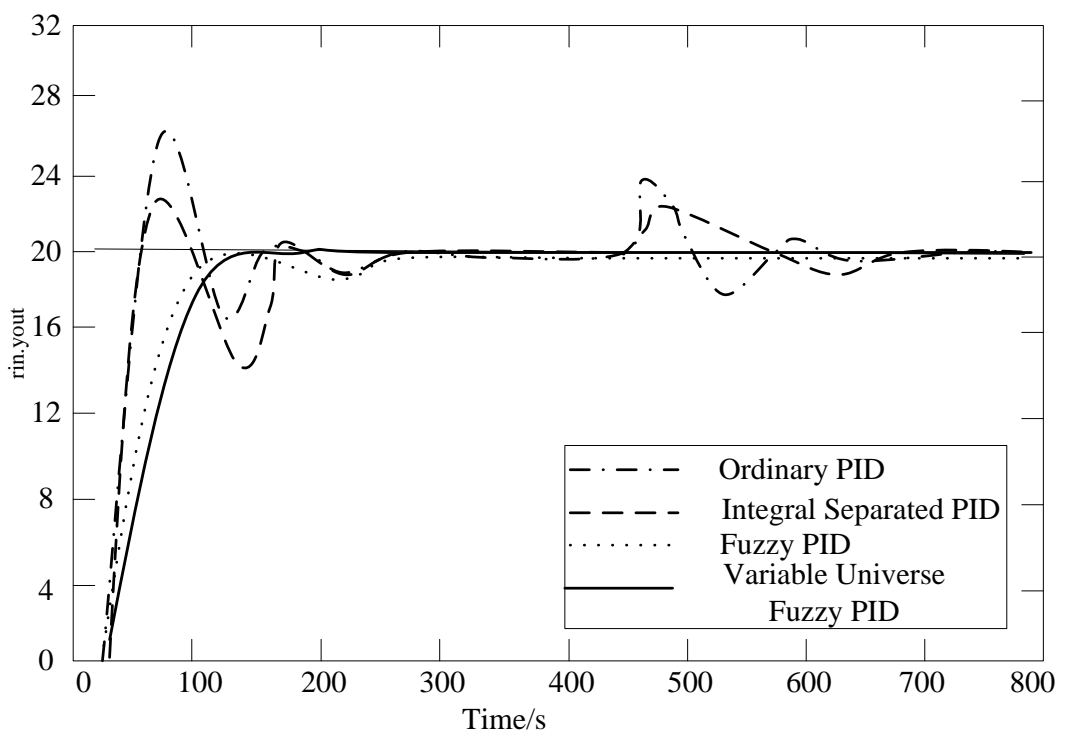

Figure 5. Comparison between Response Curves

Figure 6 is the external interrupt responding curve for four approaches at $t$ is $400 \mathrm{~s}$. It is concluded that, because of the data self-correction function of the variable universe fuzzy PID controller and fuzzy PID controller, which lead to the strong self-adaptive competence of the system, it can recover and get rid of over vibration when external interruption come. Meanwhile, self-adaptive competence of the integral separation PID control and PID control is relatively bad and will have serious vibration under similar interruption condition. 


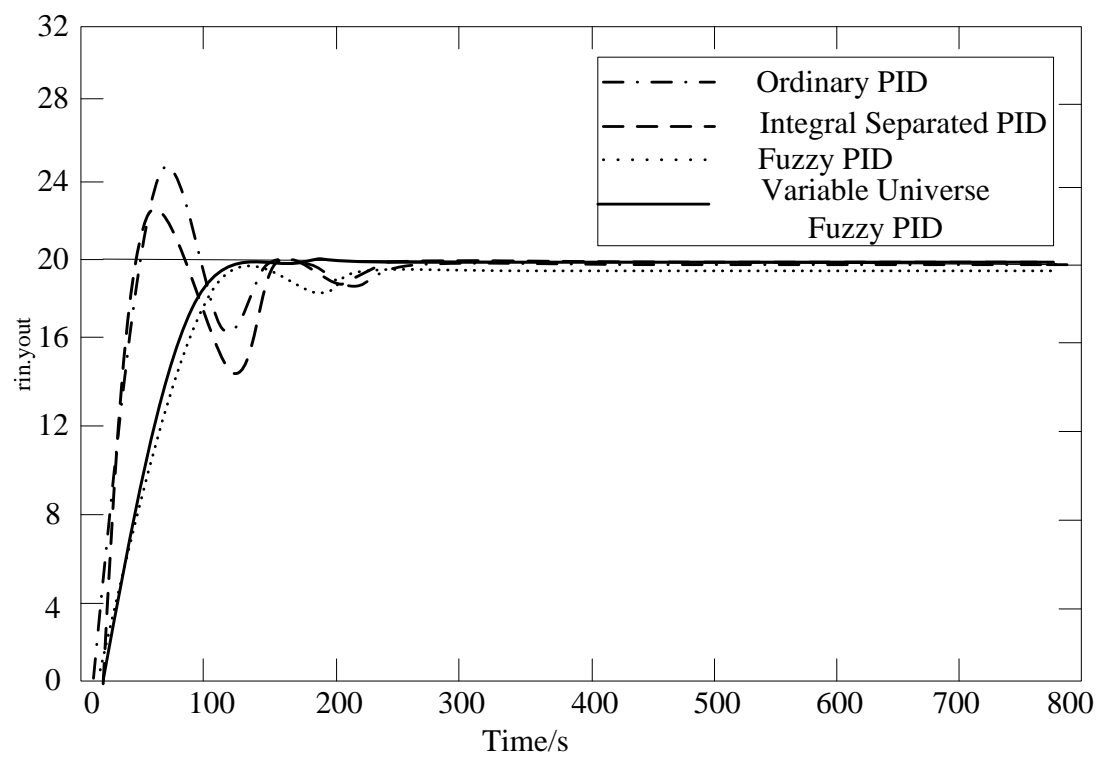

Figure 6. Comparison between Response Curves with External Interrupt

\section{Conclusions}

Adopting variable universe fuzzy PID control can not only realize self-adaptive modification of $\mathrm{K}_{\mathrm{P}}, \mathrm{K}_{\mathrm{I}}, \mathrm{K}_{\mathrm{D}}$ during the controlling process, but also modify the universe of fuzzy inference, which lead to better performance of the controller. It is concluded from the result of the stimulation that, this approach could develop the advantages of both fuzzy control and PID control, it can achieve stable and less overshoot data in less time. Meanwhile, it also obtains self-adaptive and robustness of the fuzzy PID control, which can better adaptive to the external impulse on system during the operation and is strong in antiinterference, the system has better future of industrial application.

\section{Acknowledgements}

We acknowledge support of this work by Scientific Research Foundation of National 863 Project (Grant No. 2012AA10A506-4), Youth Foundation of Northeast Agricultural University (Grant No. 2011QN201), National Natural Science Foundation (Grant No. 31101080).

The authors are grateful to the anonymous reviewers for valuable feedback.

\section{References}

[1] S. W. Tong, D. W. Qian, J. J. Fang and H. X. Li, "Integrated modeling and variable universe fuzzy control of a hydrogen-air fuel cell system", International Journal of Electrochemical Science, vol. 8, no. 3, (2013).

[2] N. k. Bachache and J.Y. Wen, "PSO and GA designed Pareto of Fuzzy Controller in AC Motor Drive", International Journal of Control and Automation, vol. 6, no. 5, (2013).

[3] S. Gao, X. X. Xu, Q. K. Xiao and Q. Pan, "Fuzzy Adaptive Control of Airborne Electro-Optical Tracking Systems Based on Variable Universe", Advanced Materials Research, vol. 383, (2012).

[4] T. Yu, W. J.Yu and Z. W. Li, "A novel optimal method of variable-universe fuzzy control based on Qlearning algorithms", Control Theory \& Applications, vol. 28, no.11, (2011).

[5] J.Wang, W. Si and H. Li, "Robust ISS-satisficing variable universe indirect fuzzy control for chaotic systems", Chaos, Solitons \& Fractals, vol.39, no.1, (2009).

[6] L. A. Zadeh, "Fuzzy sets", Information and Control, vol.8, no.3, (1965). 
[7] S. X. Zhu and C. J. Chen, "Study on Variable Universe Fuzzy PID Control for Landing Gear Based on MRF (Magneto-Rheological Fluid) Damper”, Applied Mechanics and Materials,vol.233, (2012).

[8] J. S. Liang, Y.Yuan and T. S. Zhang, "Design and Implementation of Expert Fuzzy Control System for an Environmental Chamber", Computing Technology and Automation, vol. 43, (2003).

[9] Y. Wang and Y. Lu, "Design of longitudinal predictive re-entry guidance law based on variable universe fuzzy-PI composite control", Journal of Control Theory and Applications, vol. 10, no. 2, (2012).

[10] L. Zhou, T. Yu , W. Yu and K. Wang, "A New Method of Variable Universe Fuzzy Control Based on Q Learning Algorithm", Power and Energy Engineering Conference (APPEEC), 2012 Asia-Pacific, (2012), pp.1-5.

[11] K. Tanaka and H. O. Wang. Fuzzy control systems design and analysis: a linear matrix inequality approach. John Wiley \& Sons, (2004).

[12] P. K. Wong, S. J. Huang, T. Xu, H. C. Wong and Z. C. Xie, "Design of a New Suspension System Controlled by Fuzzy-PID with Wheelbase Preview", Applied Mechanics and Materials, vol. 192, (2012).

[13] R. T. Hou, Q. Wang, J. Wang, J. J. Wang, Y. Lu and J.U. Kim, "A Fuzzy Control Method of Traffic Light with Countdown Ability", International Journal of Control and Automation, vol.5, no.4, (2012) .

[14] X. G. Duan, H. Deng and H. X. Li, "A Saturation-Based Tuning Method for Fuzzy PID Controller", Industrial Electronics, IEEE Transactions on, (2013), pp.5177-5185.

[15] G. M. Guo, and Q. Fang, "Design of Fuzzy Logic Guidance Law for Missiles using the Strategy of Variable Universe", Fire Control \& Command Control, vol.5, no.49, (2011).

[16] Z. W. Woo, H. Y. Chung and J. J. Lin, “A PID type fuzzy controller with self-tuning scaling factors”, Fuzzy Sets and Systems, vol.115, no.2, (2000).

[17] M. G. Zhang, P. Wang, Z. G. Wang and Y. Niu, "An application study of the variable universe fuzzy logic control integrated with the self-tuning PID internal model control used in a main steam temperature control system", Industrial Instrumentation \& Automation, vol. 3, no.6, (2008).

[18] H. Zheng, H. B. Xu and G. P. Zuu, "Adaptive fuzzy control based on variable universe and its application to aero engine turbine power generator", Control Theory \& Applications, vol.2, (2008).

[19] J. W.Liu, D.Wei and X.Liu, "Temperature fuzzy PID control for variable air volume system", Journal of Civil, Architectural \& Environmental Engineering, vol. 4, no.19, (2009).

[20] L. Wang and K. Zhang, "Fuzzy PID Control System Based on MATLAB", Journal of Detection \& Control, vol. 2, no. 17, (2008).

[21] J. Q. Ni, C. Vinckier and J. Hendriks, "Production of carbon dioxide in a fattening pig house under field conditions", Release from the manure atmospheric environment, vol.33, no. 22, (1999).

[22] K. K. Ahn and D. Q. Truong, "Online tuning fuzzy PID controller using robust extended Kalman filter", Journal of Process Control, vol. 19, no. 6, (2009).

[23] M. Y. Li, Y. L. Du and H. P. Jiang, "Application of intelligent self-tuning PID in temperature control system of medicament", Control Theory \& Applications, vol.5, no. 32, (2003).

\section{Authors}

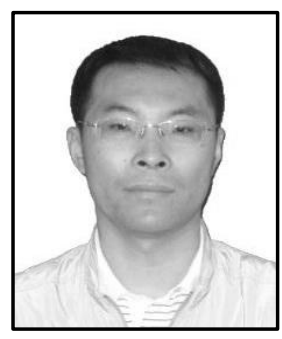

Xiao Yu was born in Harbin, china, in 1976. He received the B.S. degree in Computer Science and Technology and the M.S. degree in Computer Application Technology from School of Electrical and Information Engineering, Northeast Agricultural University, Harbin, China, in 2001, 2009, respectively, and currently pursuing the Ph.D. degree at the School of Biological and Agricultural Engineering, Jilin University, Changchun, China. He joined the Electrical and Information Engineering, Northeast Agricultural University since 2001 and currently is an Associate Professor. His research interests include Agricultural facilities environmental control and energy saving technologies and Agricultural Information Engineering. 


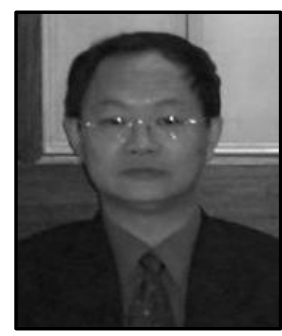

Hai-Ye $\mathbf{Y u}$ is the vice-principal of Biological and Agricultural Engineering, Jilin University, Professor, Ph.D. supervisor, Agricultural Biological Environmental and Energy Engineering doctoral academic leaders, has been engaged in agricultural robots, automatic detection and control technology research greenhouse facility. Made a number of research results in precision seeder, agricultural robots, agricultural facilities environment monitoring and intelligent management.

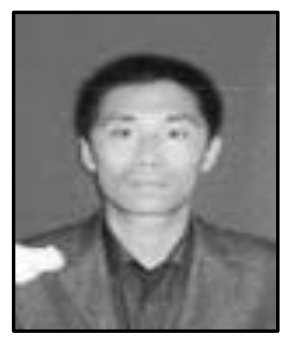

Guo-Gang Zhao is currently pursuing the Ph.D. Degree at Biological and Agricultural Engineering, Jilin University, Changchun, China. His research interest is automatic control of agricultural facilities environment. 